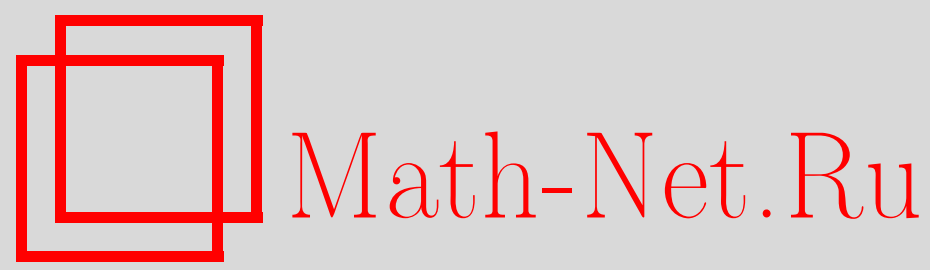

О. В. Висков, Тождество Дарбу и его аналоги, Матем. заметки, 2000, том 68, выпуск 3, 332-338

DOI: https://doi.org/10.4213/mzm950

Использование Общероссийского математического портала Math-Net.Ru подразумевает, что вы прочитали и согласны с пользовательским соглашением http://www .mathnet.ru/rus/agreement

Параметры загрузки:

IP : 3.85 .5 .30

26 апреля 2023 г., 17:53:26

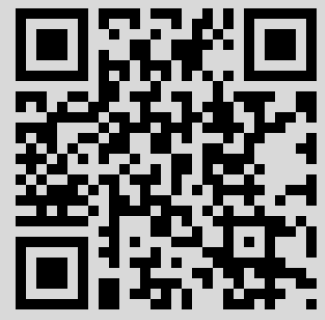




\section{ТОЖДЕСТВО ДАРБУ И ЕГО АНАЛОГИ}

\section{О.В. Висков}

Фиксируем натуральное $n$ и обозначим $F(x, y)=\sum_{m=0}^{n} f^{(n-m)}(x) g^{(m)}(y)$, где $f(\cdot)$ и $g(\cdot)$ - произвольные достаточно гладкие функции. Приводится три различных доказательства справедливости равенства

$$
F(x, y)-F(y, x)=\int_{y}^{x}\left\{f^{(n+1)}(t) g(x+y-t)-f(t) g^{(n+1)}(x+y-t)\right\} d t .
$$

Устанавливаются также дискретный и некоммутативный аналоги этого тождества.

Библиограбфия: 7 названий.

1. В ходе своих исследований, посвященных возможности распространения интегральной теоремы о среднем на комплексный случай, Дарбу существенно опирался на следующее тождество (см. [1, формула (7)]):

$$
\begin{aligned}
\varphi^{(n)}(0)\{f(z)-f(a)\}= & \sum_{m=1}^{n}(-1)^{m-1}(z-a)^{m}\left\{\varphi^{(n-m)}(1) f^{(m)}(z)-\varphi^{(n-m)}(0) f^{(m)}(a)\right\} \\
& +(-1)^{n}(z-a)^{n+1} \int_{0}^{1} \varphi(t) f^{(n+1)}(a-a t+z t) d t .
\end{aligned}
$$

Здесь $f(z)$ - функция, аналитическая во всех точках отрезка, соединяющего точки $a$ и $z$, а $\varphi(t)$ - произвольный полином степени $n$ от $t$. Именно в этой форме соотношение (1) приводится в $[2, \S 7.1]$, где оно названо формулой Дарбу.

В качестве простейшего частного случая, отвечающего выбору $\varphi(t)=(t-1)^{n}$ в $(1)$, эта формула содержит разложение Тейлора с остаточным членом в интегральной форме:

$$
f(z)=\sum_{m=0}^{n} \frac{(z-a)^{m}}{m !} f^{(m)}(a)+\int_{a}^{z} \frac{(z-t)^{n}}{n !} f^{(n+1)}(t) d t .
$$

Интересно также отметить еще один, рассмотренньй в [1], частньй случай тождества (1). Если в (1) заменить $n$ на $2 n$ и выбрать затем $\varphi(t)=t^{n}(t-1)^{n}$, то после несложных преобразований получим тождество

$$
\begin{aligned}
& \sum_{m=0}^{n} \frac{(2 n-m) ! n !}{(n-m) ! m !}(z-a)^{m}\left[(-1)^{m} f^{(m)}(z)-f^{(m)}(a)\right] \\
& \quad=\int_{0}^{1} t^{n}(t-1)^{n}(z-a)^{2 n+1} f^{(2 n+1)}(a-a t+z t) d t
\end{aligned}
$$


(см. также [2, с. 176]). Дарбу отмечает, что тождество (3) имеет преимущество перед формулой Тейлора (2), поскольку при его применении для вычисления значений данной функции ошибка приближения имеет порядок $(\Delta z)^{2 n+1}$, где $n$ - число используемых при этом производных приближаемой функции, в то время как формула Тейлора в этой ситуации гарантирует лишь порядок $(\Delta z)^{n+1}$.

Контекст, в котором Дарбу получил равенство (1), потребовал от него излишне жестких ограничений и на аппроксимируемую функцию $f(\cdot)$ (предполагалась ее аналитичность) и на аппроксимирующую последовательность $\varphi^{(m)}(\cdot), m=0,1, \ldots(\varphi(\cdot)$ считалось полиномом). В действительности же справедливо следующее утверждение.

СИММЕТРИЧНАЯ ФОРМА ТОЖДЕСТВА ДАРБУ. Для произвольныx $n+1$ paз непрерывно дифферениируемых функиий $f(\cdot)$ и $g(\cdot)$ справедливо равенство

$$
\begin{aligned}
& \sum_{m=0}^{n} f^{(n-m)}(x) g^{(m)}(y)-\sum_{m=0}^{n} f^{(n-m)}(y) g^{(m)}(x) \\
& \quad=\int_{y}^{x}\left\{f^{(n+1)}(t) g(x+y-t)-f(t) g^{(n+1)}(x+y-t)\right\} d t .
\end{aligned}
$$

Заметим сразу же, что если в этом соотношении выбрать в качестве $f(x)$ функцию $f(a+x z-a x)$, а на роль $g(x)$ определить полином $\varphi(1-x)$ степени $n$, то, как легко можно убедиться, (4) превратится в формулу Дарбу (1).

Будет приведено несколько подходов к доказательству тождества (4). Индуктивное доказательство этого тождества изложено в п. 2. Другой подход (см. п. 4) базируется на представляющем независимый интерес новом некоммутативном тождестве в алгебре Гейзенберга-Вейля. В п. 7 намечен еще один путь доказательства формулы Дарбу (4), который опирается на ключевое для этой заметки тождество (13), представляющее собой разностньй аналог равенства (4). Простые примеры использования этого аналога приведены в п. 6. Иллюстрации применения самого тождества Дарбу (4) читатель может найти в [3], [4]. Отметим также, что в [3] приводится еше одно независимое доказательство тождества (4).

2. Докажем тождество (4), используя принцип полной математической индуции по степени гладкости $n$ участвующих в нем функций. Заметим прежде всего, что

$$
\frac{d}{d t}\{f(t) g(x+y-t)\}=f^{\prime}(t) g(x+y-t)-f(t) g^{\prime}(x+y-t),
$$

и поэтому в силу формулы Барроу-Ньютона-Лейбница справедливо равенство

$$
f(x) g(y)-f(y) g(x)=\int_{y}^{x}\left\{f^{\prime}(t) g(x+y-t)-f(t) g^{\prime}(x+y-t)\right\} d t .
$$

Это тождество служит основанием доказательства по индукции симметрической формы равенства Дарбу (4), поскольку при $n=0$ (4) превращается в (5). Предполагая справедливость (4) для любых достаточно гладких функций $f(\cdot)$ и $g(\cdot)$, запишем это тождество в случае, когда роль $f(\cdot)$ играет $f^{\prime}(\cdot)$ :

$$
\begin{aligned}
& \sum_{m=0}^{n} f^{(n+1-m)}(x) g^{(m)}(y)-\sum_{m=0}^{n} f^{(n+1-m)}(y) g^{(m)}(x) \\
& \quad=\int_{y}^{x}\left\{f^{(n+2)}(t) g(x+y-t)-f^{\prime}(t) g^{(n+1)}(x+y-t)\right\} d t .
\end{aligned}
$$


Теперь воспользуемся (5), заменяя в этом тождестве функцию $g(\cdot)$ ее $(n+1)$-й производной $g^{n+1}(\cdot)$ :

$$
f(x) g^{(n+1)}(y)-f(y) g^{(n+1)}(x)=\int_{y}^{x}\left\{f^{\prime}(t) g^{(n+1)}(x+y-t)-f(t) g^{(n+2)}(x+y-t)\right\} d t .
$$

Остается только сложить равенства (6) и (7), чтобы убедиться в справедливости индуктивного перехода.

3. Равенство (4) существенно обобщает полученное в [4] тождество

$$
\sum_{m=0}^{n} \frac{a_{m}(x)}{m !} g^{(m)}(y)=\sum_{m=0}^{n} \frac{a_{m}(y)}{m !} g^{(m)}(x)+\int_{x}^{y} \frac{a_{n}(\tau)}{n !} g^{(n+1)}(x+y-\tau) d \tau
$$

которому подчиняются произвольные полиномы Аппеля $a_{n}(x), n=0,1, \ldots$ Напомним, что последовательность $a_{n}(x), n=0,1, \ldots$, полиномов $a_{n}(x)$ называется последов $a^{-}$ тельностью полиномов Аппеля, если их (формальная) экспоненциальная производящая функция имеет вид

$$
\sum_{n \geqslant 0} a_{n}(x) \frac{t^{n}}{n !}=A(t) e^{x t}
$$

где $A(t)-$ (формальньй) степенной ряд, удовлетворяющий условию $A(0) \neq 0$. Из (9) видно, что

$$
\frac{d}{d x} a_{0}(x)=0, \quad \frac{d}{d x} a_{n}(x)=n a_{n-1}(x), \quad n=1,2, \ldots,
$$

и поэтому, полагая в (4) $f(x)=a_{n}(x) / n$ ! и не накладьвая на $g(y)$ никаких других ограничений, кроме существования нужного числа производных, приходим к тождеству (8).

4. Тождество (8) было получено в [3] в качестве следствия некоммутативного тождества, которому подчиняются полиномы Аппеля в алгебре Гейзенберга-Вейля. Приведем вьвод симметричной формы тождества Дарбу (4), следуя ходу рассуждений, изложенному в [4]. Сначала докажем справедливость следующего результата.

НЕКОММУТАТИВНЫЙ АНАЛОГ ТОЖДЕСТВА ДАРБУ. Пусть әлементы $a, b$ u извольной ассоциативной алгебры связаны коммутационными соотношениями

$$
a b-b a=c, \quad a c=c a, \quad b c=c b .
$$

Тогда для любого полинома (или формального степенного ряда) $f(\cdot)$ и произвольного иелого неотрицательного п справедливо равенство

$$
b \sum_{m=0}^{n} c^{n-m} f^{(n-m)}(a) b^{m}=f(a) b^{n+1}-c^{n+1} f^{(n+1)}(a)
$$

В самом деле, индуктивньм рассуждением по степени $f(\cdot)$ легко убедиться, что из коммутационных соотношений (10) вытекает справедливость равенства

$$
f(a) b-b f(a)=c \frac{d f(a)}{d a}
$$


для произвольного $f(\cdot)$. Перебрасывая теперь в левой части (11) стоящую перед суммой образующую $b$ слева направо и используя (12), получаем “телескопическую" сумму, соседние члены которой, кроме первого и последнего (а именно эти члены и стоят в правой части (11)), взаимно уничтожаются.

Реализуем $a$ оператором умножения на $y-x$ при фиксированном $y$ и в качестве $b$ выберем оператор дифференцирования $D=d / d x$. Легко проверяется, что при выбранной реализации коммутационные соотношения (10) будут выполнятся, причем роль $c$ играет тождественный оператор. В этой ситуации тождество (11) после применения обеих его частей к $n+1$ раз дифференцируемой функции $g(x)$ дает

$$
\left(\sum_{m=0}^{n} f^{(n-m)}(y-x) g^{(m)}(x)\right)^{\prime}=f(y-x) g^{(n+1)}(x)-f^{(n+1)}(y-x) g(x) .
$$

Интегрируя это равенство и производя простые замены, приходим к доказываемому тождеству (4).

5. Обозначим через $\Delta$ разностньй оператор, сопоставляющий функции $F(x)$ целочисленного аргумента $x$ разность $F(x+1)-F(x)$, и пусть $\Delta^{k}$ - его $k$-я итерация. Справедливо следующее утверждение.

РАЗНОСТНЫЙ АНАЛОГ ТОЖДЕСТВА ДАРБУ. Пусть $F(\cdot) u G(\cdot)-$ произвольные функции иелочисленного аргумента. Тогда для произвольного иелого неотрицательного п и любых челых $\alpha$ и $\beta$ справедливо равенство

$$
\begin{aligned}
\sum_{m=0}^{n} & \Delta^{n-m} F(\alpha) \Delta^{m} G(\beta)-\sum_{m=0}^{n} \Delta^{n-m} F(\beta) \Delta^{m} G(\alpha) \\
& =\sum_{k=\alpha}^{\beta-1}\left\{F(\alpha+\beta-k-1) \Delta^{n+1} G(k)-\Delta^{n+1} F(\alpha+\beta-k-1) G(k)\right\} .
\end{aligned}
$$

Для доказательства справедливости (13) запишем сначала очевидное “телескопическое" равенство

$$
F(\alpha) G(\beta)-F(\beta) G(\alpha)=\sum_{k=\alpha}^{\beta-1}\{F(\alpha+\beta-k-1) G(k+1)-F(\alpha+\beta-k) G(x)\}
$$

и заметим, что его можно переписать в виде

$$
F(\alpha) G(\beta)-F(\beta) G(\alpha)=\sum_{k=\alpha}^{\beta-1}\{F(\alpha+\beta-k-1) \Delta G(k)-\Delta F(\alpha+\beta-k-1) G(k)\} .
$$

$\Phi$ иксируем неотрицательные целые числа $n$ и $m \leqslant n$. Если воспользоваться (14), подставив в это соотношение вместо функций $F(\alpha)$ и $G(\beta)$ соответственно $\Delta^{n-m} F(\alpha)$ и $\Delta^{m} G(\beta)$, то приходим к равенству

$$
\begin{array}{rl}
\Delta^{n-m} & F(\alpha) \Delta^{m} G(\beta)-\Delta^{n-m} F(\beta) \Delta^{m} G(\alpha) \\
& =\sum_{k=\alpha}^{\beta-1}\left\{\Delta^{n-m} F(\alpha+\beta-k-1) \Delta^{m+1} G(k)-\Delta^{n-m+1} F(\alpha+\beta-k-1) \Delta^{m} G(k)\right\} .
\end{array}
$$

Остается просуммировать (15) по $m$ от 0 до $n$ и заметить, что слагаемые, стоящие справа (исключая члены, отвечающие $m=0$ в первой сумме и $m=n$ во второй), будут взаимно уничтожать друг друга. В результате приходим к желаемому равенству (13). 
6. Соотношение (13) может служить полезным инструментом для получения разложения функции целочисленного аргумента по полиномам, являющимся разностным аналогом полиномов Аппеля [5]. Эти полиномы $a_{n}(x), n=0,1, \ldots$, проще всего определить, используя их (формальную) экспоненциальную производящую функцию:

$$
\sum_{n \geqslant 0} a_{n}(x) \frac{t^{n}}{n !}=A(t)(1+t)^{x},
$$

где $A(t)$ - (формальный) степенной ряд, удовлетворяющий условию $A(0) \neq 0$.

Простейшим примером таких полиномов являются так назьваемые убывающие факториалы

$$
a_{0}(x)=1, \quad a_{n}(x) \equiv(x)_{n}=x(x-1) \cdots(x-n+1), \quad n=1,2, \ldots,
$$

определяемые $(16)$ с $A(t)=1$. Следует упомянуть также полиномы Пуассона-Шарлье с параметром $\lambda$ :

$$
\pi_{n}(x)=\sum_{m=0}^{n}\left(\begin{array}{l}
n \\
m
\end{array}\right)(x)_{m}(-\lambda)^{n-m}, \quad n=0,1, \ldots,
$$

экпоненциальная производящая функция которых задается (16) с $A(t)=\exp (-\lambda t)$ (см. [6, соотношение 2.61.2]).

Заметим, что если применить разностньй оператор $\Delta$ к обеим частям равенства (16) и после этого сравнить коэффициенты при одинаковых степенях $t$, то придем к равенствам

$$
\Delta a_{0}(x)=0, \quad \Delta a_{n}(x)=n a_{n-1}(x), \quad n=1,2, \ldots
$$

Легко понять, что, в свою очередь, из (19) следует соотношение (16) для некоторого формального степенного ряда $A(t)$, так что равенство (16) можно рассматривать как альтернативное определение рассматриваемого класса полиномов. Соотношение (19) показывает, что по отношению к операции взятия конечной разности эти полиномы ведут себя подобно убывающим факториалам (17). В частности, из (16) вытекает, что

$$
\Delta^{m} a_{n}(x)=\left(\begin{array}{c}
n \\
m
\end{array}\right) m ! a_{n-m}(x)
$$

для любого целого $m, 0 \leqslant m \leqslant n$.

Отметим еще, что из условия $A(0) \neq 0$ в (16) вытекает, что степень полинома $a_{n}(x)$ равна $n$, и поэтому с каждой последовательностью рассматриваемых полиномов можно связать линейньй функционал $E_{a}$, однозначно определяемый соотношениями

$$
E_{a}\left[a_{n}(x)\right]= \begin{cases}1, & \text { если } n=0, \\ 0, & \text { если } n \neq 0 .\end{cases}
$$

Например, для убьвающих факториалов (17) линейный функционал $E_{a}$ сопоставляет любому полиному $p(x)$ его значение при $x=0$. Что касается полиномов Пуассона-Шарлье (18), то для положительного значения параметра $\lambda$ функционал (21) является осреднением по пуассоновскому распределению с параметром $\lambda$, т.е. в этом случае

$$
E_{\pi}[p(x)]=\sum_{k=0}^{\infty} p(k) \frac{\lambda^{k}}{k !} \exp (-\lambda)
$$


Вернемся теперь к соотношению (13) и выберем в нем $F(x)=a_{n}(x) / n !$, где последовательность $a_{n}(x), n=0,1, \ldots$, определяется (16) (или (19)), а $G(x)$ попрежднему будем считать произвольной функцией целочисленного аргумента. При таком выборе из равенства (20) вытекает, что разностньй аналог тождества Дарбу (13) принимает вид

$$
\sum_{m=0}^{n} \frac{a_{m}(\alpha)}{m !} \Delta^{m} G(\beta)-\sum_{m=0}^{n} \frac{a_{m}(\beta)}{m !} \Delta^{m} G(\alpha)=\sum_{k=\alpha}^{\beta-1} \frac{a_{n}(\alpha+\beta-k-1)}{n !} \Delta^{n+1} G(k) .
$$

Более привьчную форму симметричное тождество (23) принимает после применения к обеим его частям линейного функционала (21) по переменной $\alpha=x$ :

$$
G(\beta)=\sum_{m=0}^{n} \frac{a_{m}(\beta)}{m !} E_{a} \Delta^{m} G(x)+E_{a} \sum_{k=x}^{\beta-1} \frac{a_{n}(x+\beta-k-1)}{n !} \Delta^{n+1} G(k) .
$$

Поскольку, как уже отмечалось, для полиномов (17) $E_{a} p(x)=p(0)$, последнее равенство превращается в формулу Ньютона (см. [7]) “с остаточньм членом"

$$
G(\beta)=\sum_{m=0}^{n}\left(\begin{array}{c}
\beta \\
m
\end{array}\right) \Delta^{m} G(0)+\sum_{k=0}^{\beta-1}\left(\begin{array}{c}
\beta-k-1 \\
n
\end{array}\right) \Delta^{n+1} G(k)
$$

Другой подход к доказательству этого равенства, основанньй на некоммутативном тождестве, можно найти в [4].

Для полиномов Пуассона-Шарлье (18) тождество (24) дает

$$
G(\beta)=\sum_{m=0}^{n} \frac{\pi_{m}(\beta)}{m !} E_{\pi} \Delta^{m} G(x)+E_{\pi} \sum_{k=x}^{\beta-1} \frac{\pi_{n}(x+\beta-k-1)}{n !} \Delta^{n+1} G(k),
$$

где, как было отмечено, линейный функционал $E_{\pi}$ задается $(22)$.

Можно показать (используя, например, разностную формулу Родрига для полиномов Пуассона-Шарлье), что коэффициенты этого разложения лишь формой записи отличаются от обычных коэффициентов разложения по ортогональным полиномам. Однако неясно, каким образом в рамках теории ортогональных полиномов можно получить явный вид "остаточного члена".

7. Воспользуемся разностным аналогом (13) тождества Дарбу для альтернативного доказательства справедливости самого тождества Дарбу (4). С этой целью рассмотрим две $n+1$ раз непрерьвно дифференцируемые функции $f(\cdot)$ и $g(\cdot)$ и фиксируем переменные $x$ и $y$. Выберем натуральное число $N$ и для произвольного целого $k$ обозначим $x_{k}=x+k h$, где $h=(y-x) / N$. Используя тождество (13) для функций целочисленного аргумента $F(\cdot)$ и $G(\cdot)$, определенных соотношениями

$$
F(k)=f\left(x_{k}\right), \quad G(k)=g\left(x_{k}\right),
$$


и полагая в этом равенстве $\alpha=0, \beta=N$, видим, что

$$
\begin{aligned}
& \sum_{m=0}^{n} \Delta^{n-m} F(0) \Delta^{m} G(N)-\sum_{m=0}^{n} \Delta^{n-m} F(N) \Delta^{m} G(0) \\
& \quad=\sum_{k=0}^{N-1}\left\{F(N-k-1) \Delta^{n+1} G(k)-\Delta^{n+1} F(N-k-1) G(k)\right\} .
\end{aligned}
$$

В обозначениях (27) с учетом очевидных равенств

$$
x_{0}=x, \quad x_{N}=y, \quad x_{N-k-1}=x+y-x_{k+1}
$$

тождество (28) можно переписать в виде

$$
\begin{aligned}
& \sum_{m=0}^{n} \Delta_{h}^{n-m} f(x) \Delta_{h}^{m} g(y)-\sum_{m=0}^{n} \Delta_{h}^{n-m} f(y) \Delta_{h}^{m} g(x) \\
& \quad=\sum_{k=0}^{N-1}\left\{f\left(x+y-x_{k+1}\right) \Delta_{h}^{n+1} g\left(x_{k}\right)-\Delta_{h}^{n+1} f\left(x+y-x_{k+1}\right) g\left(x_{k}\right)\right\},
\end{aligned}
$$

где $\Delta_{h}$ - оператор, сопоставляющий функции $f(x)$ разность $f(x+h)-f(x)$, и $\Delta_{h}^{k}-$ его $k$-я итерация. Разделим обе части равенства $(29)$ на $h^{n}$ и перепишем его в виде

$$
\begin{aligned}
& \sum_{m=0}^{n}\left(\frac{\Delta_{h}}{h}\right)^{n-m} f(x)\left(\frac{\Delta_{h}}{h}\right)^{m} g(y)-\sum_{m=0}^{n}\left(\frac{\Delta_{h}}{h}\right)^{n-m} f(y)\left(\frac{\Delta_{h}}{h}\right)^{m} g(x) \\
& \quad=h \sum_{k=0}^{N-1}\left\{f\left(x+y-x_{k+1}\right)\left(\frac{\Delta_{h}}{h}\right)^{n+1} g\left(x_{k}\right)-\left(\frac{\Delta_{h}}{h}\right)^{n+1} f\left(x+y-x_{k+1}\right) g\left(x_{k}\right)\right\} .
\end{aligned}
$$

Остается заметить, что $h$ стремится к нулю с ростом $N$, и поэтому равенство (30) в пределе (при $N \rightarrow \infty$ ) превращается в $(4)$, поскольку разностньй оператор $\Delta_{h} / h$ переходит в пределе в оператор дифференцирования, а правая часть (30) в этих условиях стремится к интегралу, стоящему в правой части (4).

Автор признателен анонимному рецензенту, замечания которого способствовали улучшению первоначального варианта заметки.

\section{СПИСОК ЦИТИРОВАННОЙ ЛИТЕРАТУРЫ}

[1] Darboux G. Sur les développements en série des fonctions d'une seule variable // J. de Math. 1876. V. 3. № 2. P. 291-313.

[2] Уиттекер Э. Т., Ватсон Дж. Н. Курс современного анализа. М.: Физматгиз, 1963.

[3] Висков О.В. О формуле Дарбу // Интегральные преобразования и специальные функции. Информационный бюллетень. 1998. Т. 1(3). С. 2-5.

[4] Висков О.В.Некоммутативное тождество для полиномов Аппеля // Матем. заметки. 1998. T. 64. № 2. C. 307-311.

[5] Boas R.P., Buck R. C., Creighton R. Polynomial Expansions of Analytical Functions. Berlin: Springer Verlag, 1964.

[6] Сегё Г. Ортогональные многочлены. М.: Физматгиз, 1962.

[7] Гельфонд А. О. Исчисление конечных разностей. М.: Физматгиз, 1959. 